\title{
Identification of Potential Maintainer and Restorer Lines Using Testcross Hybrids
}

\author{
Mohammad Amir Hossain', Md. Jamil Hasan², Mosammat Umma Kulsum², \\ Md. Mahathir Sarker ${ }^{3 *}$
}

${ }^{1}$ Plant Breeding Division, Bangladesh Rice Research Institute, Bangladesh-1701

${ }^{2}$ Hybrid Breeding Division, Bangladesh Rice Research Institute, Bangladesh-1701

${ }^{3}$ Department of Genetics and Plant Breeding, EXIM Bank Agricultural University, Bangladesh-6300

\section{A R T I C L E I N F O}

\section{Research Article}

Received 23 August 2017

Accepted 07 May 2018

Keywords:

CMS lines

Restorers

Maintainers

Rice

Cytoplasmic Male Sterility

*Corresponding Author:

E-mail: mahathir.sarker@yahoo.com

A B S T R A C T

Five CMS lines were crossed with 49 genotypes as 'testers' to get 245 hybrids. The 245 hybrids were subjected to pollen and spikelet fertility analysis. Among the 245 hybrids 21 hybrids were expressed as restorers, 24 as maintainers, and 200 intermediate types. Six tester's viz., BR6592-4-6-4, BR6839-41-5-1, IR74052-184-3-3, IR72593-B-3-2-2-2, BR7011-37-1-2, and IR72049-B-R-22-3-1-1 were identified as restorers for highest 2 lines out of the five CMS lines, differently. None of them were found to be restorer for all the five CMS lines. BAU581 was found to be maintainer for four lines except for D.ShanA. Purbachi was found to be maintainer for three lines except for D.ShanA and IR73328A out of the five CMS lines. Out of 245 crosses only 45 crosses contributed directly to the identification of maintainer and restorer. Other crosses were more or, less of intermediate types which indicated neither maintainer nor restorer. It is well known that, pollen fertility is controlled by one dominant gene $(R f R f)$. If pollen fertility is governed by only one gene the product would be either restorer or, maintainer where will be no existence of intermediate types. So, there might be modifier genes in different genotypes which interacted with male sterile nuclear genes that resulted in intermediate male sterility in the crosses.

DOI: https://doi.org/10.24925/turjaf.v6i8.953-962.1481

\section{Introduction}

Increased and sustainable rice production must be achieved in less land in near future due to higher population pressure on ever decreasing resources (Rothschild, 1997). So, there is no other alternative but to increase per unit production. But the yield potential of modern rice varieties reached the plateau. Under such situation, alternative approaches should be implemented for development and adoption of yield enhancing technologies. Presence of hybrid vigor and availability of pollination control mechanism such as male sterility in crop plants has been proved to be an efficient tool for obtaining higher returns in advance agricultural economy (Hawlader et al., 1997). Use of male sterility system would be appropriate approach for commercial exploitation of heterosis in rice. Though, several male sterility systems are known to occur in rice, of which cytoplasmic genetic male sterility (CMS) has been widely used for developing rice hybrids. CMS system is controlled by the interaction of cytoplasmic genes with nuclear genes (Kaul, 1997). Development of a hybrid rice using CMS requires development of a male sterile line (A-line), a maintainer line (B-line) and a restorer line (R- line). Where, A-line is male sterile line which is used as female line in commercial hybrid production plot; B-line is isogenic line of A-line which is needed to maintain Aline; and R-line is male fertile line which is used as pollen parent in commercial seed production plot. B-line is considered as key line without which A-line cannot be maintained. Test cross programs help to identify maintainers as well as restorers. Maintainer lines are converted into new CMS lines and restorer lines are subsequently used as male parent in hybrid development program. IRRI initiated hybrid rice program in Bangladesh in late eighties through BRRI. But the program did not gain momentum. CMS lines introduced from elsewhere were not well adapted to the given target area. Successful use of hybrid vigor in rice largely depends on availability of locally developed cytoplasmic genetic male sterile (CMS) and restorer lines (Kumar et al. 1996). Julfiquar et al. (2002) evaluated some Chinese CMS lines e.g. V20A and Zhen Shan97A along with their maintainer for adaptability and performance but those were not adaptable to Bangladesh condition as they become highly susceptible to disease and insects. 
Moreover, breeders need wide range of CMS lines to produce desired hybrids. Therefore, it is necessary to transfer available CMS system into local elite breeding lines. So, use of local CMS line would be helpful to alleviate this problem and to develop adaptable, heterotic hybrids. Kumar et al. (1996) identified two effective maintainers for WA cytoplasmic source and successfully converted them into local lines through backcrossing. But it is not complete process in searching maintainer and restorer lines from rice elite breeding lines against different local and exotic cytosources. So, the present investigation was undertaken with the following objectives: to identify maintainers and restorers from elite lines based on test-cross pollen performance and to initiate backcrossing for conversion of maintainers into new CMS lines.

\section{Materials and Methods}

Five alien male sterile lines (IR58025A, BRRI1A, D.ShanA, BRRI9A and IR73328A) and 49 elite lines were grown in the field during Boro season. Three sets of elite lines were sown in 5 days interval. Similarly, four sets of CMS lines were sown in 5, 3 and 4 days interval, respectively. First set of elite lines was 5 days earlier than the first set of CMS lines. Second and third set of elite lines were sown on the same date of sowing of first and second set of CMS lines, respectively. Unit plot size for each CMS and elite lines was $2.72 \mathrm{~m}^{2}(3.4 \mathrm{~m} \times 0.8 \mathrm{~m})$. Twenty one (21) days old seedlings of the tested entries were transplanted in separate strips with spacing of $20 \mathrm{~cm}$ between plant to plant and $20 \mathrm{~cm}$ between row to row. Unit plot consists of 68 plants. Adequate soil fertility was ensured by applying Urea-TSP-MP-Gypsum- $\mathrm{ZnSO}_{4}$ at the rate of 150-100-70-60-10 kg/ha, respectively during $\mathrm{T}$. Aman season. All the TSP, MP, Gypsum and $\mathrm{ZnSO}_{4}$ were applied during final land preparation. Total Urea was applied in three installments, at 15 days after transplanting (DAT), 30 DAT, and 45 DAT. Necessary intercultural operations were carried out during the cropping period for proper growth and development of plants. For facilitating hybridization, at flowering stage, several sterile plants from CMS lines were transferred in pots which were previously filled with soil and crossed with pollens from plants of 49 elite lines (Table 1). Clipping of CMS panicles was done in the afternoon and hand pollination was carried out in the following morning by dusting pollen from selected elite lines. Adequate measures were taken to avoid undesirable pollination. The clipped and crossed panicles were tagged and bagged properly with white and brown papers, respectively. After pollination, bagging continued for 3-4 days to avoid unwanted pollination. After maturity sufficient number of spikelet from panicles of 245 crosses were collected. Properly dried and cool seeds were kept in store for further study. All $\mathrm{F}_{1} \mathrm{~s}$ along with their pollen parents were grown during both T. Aman and Boro seasons for testing pollen viability status of $F_{1} s$. Around, 20 spikelet were randomly selected which were collected from newly emerged panicle of $\mathrm{F}_{1}$ plant in a vial containing $70 \%$ ethanol for pollen viability test. At laboratory, one drop of $1 \%$ Iodine Potassium Iodide (IKI) stain (the stain is prepared by dissolving $1 \mathrm{~g}$ Iodine and $2 \mathrm{~g}$ Potassium iodide in $100 \mathrm{ml}$ water) was put on a glass slide.

Table 1 List of elite lines used for identifying maintainer and restorer against five alien CMS lines (IR58025A, BRRI1A, D.ShanA, BRRI9A, and IR73328A)

\begin{tabular}{|c|c|c|}
\hline $\begin{array}{l}\text { Sl. } \\
\text { No. }\end{array}$ & Genotypes & BRRI* \\
\hline 1. & BR368-15-2-2-3 & 3801 \\
\hline 2. & BR601-1-3-3-4-2-4 & 3802 \\
\hline 3. & BR5563-3-3-4-1 & dhan 48 \\
\hline 4. & BR6592-4-6-4 & 7101 \\
\hline 5. & BR6723-1-1-2 & - \\
\hline 6. & BR6839-41-5-1 & - \\
\hline 7. & BR7011-37-1-2 & - \\
\hline 8. & BAU553 & BAU \\
\hline 9. & BAU572 & BAU \\
\hline 10. & BAU581 & BAU \\
\hline 11. & IR7676-12-1-2 & 3750 \\
\hline 12. & IR9202-36-3-2 & 3756 \\
\hline 13. & IR25924-51-2-3 & 3792 \\
\hline 14. & IR61228-3B-10-3-2 & - \\
\hline 15. & IR72049-B-R-22-3-1-1 & 5917 \\
\hline 16. & IR74052-184-3-3 & - \\
\hline 17. & OM1490 & 5110 \\
\hline 18. & BKNLR-75091-GNT-B3-RST-48-2-2 & 3832 \\
\hline 19. & Moroberekan & - \\
\hline 20. & Hare Bare & 3378 \\
\hline 21. & Koshinihisini & 3397 \\
\hline 22. & Yamabiko & 3383 \\
\hline 23. & Sweon-287 & 3973 \\
\hline 24. & Hunen Wase & 3348 \\
\hline 25. & Aokaze & 3395 \\
\hline 26. & Aki Hi Kari & 3338 \\
\hline 27. & Kogahikari & 3399 \\
\hline 28. & Chinese Variety 2 & 3405 \\
\hline 29. & Chinese Variety 5 & 3419 \\
\hline 30. & Chinese Variety 8 & 3409 \\
\hline 31. & Purbachi & 6207 \\
\hline 32. & BR7-51(78)-3 & 3759 \\
\hline 33. & BR11-54-1-1 & 3970 \\
\hline 34. & BR220-1-1-1 & 3748 \\
\hline 35. & BR707C-MR-137 & 3771 \\
\hline 36. & BR7109-5R-2 & - \\
\hline 37. & BR7109-5R-4 & - \\
\hline 38. & BRRI dhan29-SC3-27 & 4410 \\
\hline 39. & IR1544-38-2-2 & 3971 \\
\hline 40. & IR11248-148-3-2-33 & 3770 \\
\hline 41. & IR19660-73-4-2 & 3968 \\
\hline 42. & IR19661-13 & 3966 \\
\hline 43. & IR69515-KKN-4-UBN-4-2-1-1 & - \\
\hline 44. & IR72022-462-3-3-2 & - \\
\hline 45. & IR72593-B-3-2-2-2 & - \\
\hline 46. & IR74963-262-5-1-3-3 & - \\
\hline 47. & IR77034-B-4-3-3 & - \\
\hline 48. & IR9447-P1-P2-4B & 3751 \\
\hline 49. & IR9708-5-1-2 & 3826 \\
\hline
\end{tabular}

* BRRI Accession No./Variety/ Source

All anthers of 5 to 6 spikelet were then taken out with the help of forceps and placed on stain of the glass slide. The anthers were crushed gently by using needle to release pollen grains. After removing debris, a cover slip was placed on crushed grains and pollen fertility status was observed under a compound microscope. The entire slide was scanned and pollen fertility was counted in three random fields. The pollen grains were classified based on 
their shape, size and extent of staining. Only dark stained round pollens were classified as fertile while unstained withered, unstained spherical and light stained round were denoted as sterile. Only two characters were recorded to identify maintainers and restorers of CMS lines. Those were pollen sterility percentage and spikelet fertility percentage.

Pollen sterility (\%): Sterile pollen of a plant at flowering stage was counted under compound microscope stained with $1 \%$ IKI solution from three random foci. Percentage of sterile pollen to the total pollen under the focus was calculated.

Spikelet fertility (\%): The proportion of filled grains to total spikelets of 10 or all (which had less than 10 panicles per hill) panicles was expressed in percentage and average was taken. $F_{1} \mathrm{~s}$ were classified as described in Table 2, based on the extent of pollen sterility (Virmani et. al., 1997).

Table 2 Pollen fertility category

\begin{tabular}{c|l}
\hline Pollen sterility $(\%)$ & \multicolumn{1}{c}{ Category } \\
\hline 100 & Completely sterile (CS) \\
$91-99$ & Sterile (S) \\
$71-90$ & Partially sterile (PS) \\
$31-70$ & Partially fertile (PF) \\
$21-30$ & Fertile (F) \\
$0-20$ & Fully fertile (FF) \\
\hline
\end{tabular}

\section{Results and Discussion}

Considering results of two T. Aman and two Boro season on pollen sterility and spikelet fertility percentage of $\mathrm{F}_{1} \mathrm{~S}$ were classified.

\section{Identification of Maintainer and Restorer Lines Against IR58025A}

Pollen sterility and spikelet fertility of forty nine crosses between IR58025A and 49 rice elite breeding lines were determined in T. Aman/2009 and Boro/200910 seasons for identification of maintainer and restorer lines from elite breeding lines of rice. Pollen sterility in $\mathrm{T}$. Aman/2009 ranged from 15.73\% (IR58025A $\times$ BR683941-5-1) to $100 \%$ (IR58025A $\times$ BAU572, IR58025A $\times$ BAU581, and IR58025A $\times$ Purbachi) and the highest spikelet fertility of the crosses against IR58025A was 74.15\% (IR58025A × BKNLR-75091-GNT-B3-RST-482-2) in T. Aman/2009 season (Table 3). Considering the results of two seasons, three crosses might be denoted as completely sterile, eight as sterile, thirteen as partially sterile, fourteen as partially fertile, seven as fertile and four as fully fertile crosses (Table 8 ). The $F_{1}$ s, IR58025A $\times$ BAU572, IR58025A $\times$ BAU581, and IR58025A $\times$ Purbachi showed $100 \%$ pollen sterility and less than $5 \%$ spikelet fertility. Such results indicated that the pollen parents (BAU572, BAU581, and Purbachi) carry maintainer genes. So, BAU572, BAU581, and Purbachi might be denoted as maintainer lines against IR58025A (Table 9). Efforts could be made to convert these $F_{1} s$ $($ IR58025A $\times$ BAU572, IR58025A $\times$ BAU581, and IR58025A $\times$ Purbachi) into local cyto-sterile lines in the background of IR58025A through backcrossing technique. In an experiment, Zaman et al. (1998) found Pusa Basmati-1 as a perfect maintainer of wild abortive
(WA) cyto-sterility from test-crosses of various Basmati lines with IR58025A. Ali et al. (2006) identified five suitable maintainer lines through test-crossing with IR58025A. On the other hand, $F_{1}$ s involving BR6592-4-64, BR6839-41-5-1, IR74052-184-3-3 and BKNLR-75091GNT-B3-RST-48-2-2 produced more than $80 \%$ pollen and spikelet fertility in both T. Aman/2009 and Boro/2009-10 seasons indicating that these pollen parents carry restorer genes against IR58025A cytoplasm and BR6592-4-6-4, BR6839-41-5-1, IR74052-184-3-3 and BKNLR-75091-GNT-B3-RST-48-2-2 might be denoted as restorer lines against IR58025A (Table 10). The crosses of IR58025A with restorers could be evaluated for heterosis to develop hybrid rice. Hoan et al. (1998) identified RPW20001 and DRGL30090 as two restorer lines against the CMS line MS577A. Other crosses showed variable percentage of pollen sterility and spikelet fertility indicating that involving pollen parents were identified as heterozygous for sterility or, fertility. Forty three potential maintainers and 10 effective restorers were identified from 264 testcross $\mathrm{F}_{1} \mathrm{~s}$ using 11 CMS lines including D.ShanA, IR58025A, BRRI1A and BRRI9A during T. Aman, 2007 (Anon, 2010). Ingale et al. (2008) also found 40 restorers and 77 maintainers among 145 genotypes for 10 CMS lines.

Identification of Maintainer and Restorer Lines Against BRRIIA

Pollen sterility of 49 crosses between CMS line BRRI1A and rice elite breeding lines ranged from 11.98\% $($ BRRI1A $\times$ IR72593-B-3-2-2-2) to $100 \%(B R R I 1 A \times$ BAU553, BRRI1A $\times$ BAU581, BRRI1A $\times$ Sweon-287, BRRI1A $\times$ Aokaze, BRRI1A $\times$ Aki Hi Kari, BRRI1A $\times$ Kogahikari, and BRRI1A $\times$ Purbachi). Likewise, spikelet fertility was ranged from $1.33 \%(B R R I 1 \mathrm{~A} \times$ Purbachi) to 83.82\% (BRRI1A $\times$ BR5563-3-3-4-1) in T Aman/2009 season (Table 4). Evaluating the results, seven crosses were identified as completely sterile, five as sterile, fourteen as partial sterile, thirteen as partial fertile, seven as fertile, and three as fully fertile (Table 8). Pollen parents (BAU553, BAU581, Sweon-287, Aokaze, Aki Hi Kari, Kogahikari and Purbachi) of complete sterile crosses were identified as potential maintainers against BRRI1A (Table 9). The completely sterile crosses could be converted into CMS lines with background of BRRI1A cytoplasm by repeated backcrossing with respective pollen parents as recurrent parent. Male parents (BR55633-3-4-1, IR72593-B-3-2-2-2, and IR9708-5-1-2) of full fertile crosses were identified as restorers against BRRI1A (Table 10). Forty three potential maintainers and 10 effective restorers were identified from 264 testcross $\mathrm{F}_{1} \mathrm{~s}$ using $11 \mathrm{CMS}$ lines including D.ShanA, IR58025A, BRRI1A, and BRRI9A during T. Aman/2007 (Anon, 2010). Again, 2 potential maintainers and 4 restorers were identified from 213 testcrosses using 59 CMS lines including D.ShanA, IR73328A, BRRI1A and BRRI9A during Boro, 2007-08 (Anon, 2010). Borkakati and Chetia (2000) found Satava as a potential maintainer for IR62829A. Singh et al. (2000) identified Sarjoo52, Saket4, IR24, and Narendra118 as restorers against IR62829A. 
Table 3 Sterility maintenance and fertility restoration ability of 49 elite lines against alien CMS line, IR58025A

\begin{tabular}{|c|c|c|c|c|c|c|c|}
\hline \multirow{2}{*}{ Sl. No. } & \multirow{2}{*}{ Pollen parents } & \multicolumn{2}{|c|}{ Pollen sterility $(\%)$ of $\mathrm{F}_{1}$} & \multicolumn{2}{|c|}{ Spikelet fertility $(\%)$ of $\mathrm{F}_{1}$} & \multirow[t]{2}{*}{ Status } & \multirow[t]{2}{*}{ Remarks } \\
\hline & & T.Aman/09 & Boro/09-10 & T Aman/09 & Boro/09-10 & & \\
\hline 1. & BR368-15-2-2-3 & 93.07 & 91.32 & 6.79 & 8.75 & $\mathrm{~S}$ & \\
\hline 2. & BR601-1-3-3-4-2-4 & 82.32 & 79.65 & 22.63 & 27.95 & PS & \\
\hline 3. & BR5563-3-3-4-1 (BRRI dhan48) & 95.48 & 93.22 & 8.50 & 11.29 & $\mathrm{~S}$ & \\
\hline 4. & BR6592-4-6-4 (BRRI dhan49) & 17.03 & 14.75 & 81.37 & 84.30 & FF & Restorer \\
\hline 5. & BR6723-1-1-2 & 24.95 & 22.72 & 54.30 & 63.97 & $\mathrm{~F}$ & \\
\hline 6. & BR6839-41-5-1 & 15.73 & 12.67 & 80.89 & 83.35 & FF & Restorer \\
\hline 7. & BR7011-37-1-2 & 26.02 & 23.79 & 57.30 & 64.20 & $\mathrm{~F}$ & \\
\hline 8. & BAU553 & 70.00 & 67.83 & 49.10 & 57.89 & PF & \\
\hline 9. & BAU572 & 100.00 & 100.00 & 1.90 & 3.45 & CS & Maintainer \\
\hline 10. & BAU581 & 100.00 & 100.00 & 2.53 & 4.95 & $\mathrm{CS}$ & Maintainer \\
\hline 11. & IR7676-12-1-2 & 57.49 & 53.73 & 42.87 & 51.21 & PF & \\
\hline 12. & IR9202-36-3-2 & 80.32 & 75.42 & 20.50 & 24.25 & PS & \\
\hline 13. & IR25924-51-2-3 & 47.92 & 44.75 & 38.21 & 46.25 & $\mathrm{PF}$ & \\
\hline 14. & IR61228-3B-10-3-2 & 81.64 & 78.53 & 21.39 & 29.72 & PS & \\
\hline 15. & IR72049-B-R-22-3-1-1 & 65.47 & 60.36 & 46.63 & 55.10 & $\mathrm{PF}$ & \\
\hline 16. & IR74052-184-3-3 & 18.09 & 15.73 & 82.98 & 85.69 & FF & Restorer \\
\hline 17. & OM1490 & 27.93 & 23.47 & 58.07 & 64.23 & $\mathrm{~F}$ & \\
\hline 18. & BKNLR-75091-GNT-B3-RST-48-2-2 & 18.78 & 16.05 & 83.15 & 86.91 & FF & Restorer \\
\hline 19. & Moroberekan & 29.30 & 26.72 & 60.12 & 68.85 & $\mathrm{~F}$ & \\
\hline 20. & Hare Bare & 94.82 & 92.35 & 6.84 & 9.75 & $\mathrm{~S}$ & \\
\hline 21. & Koshinihisini & 68.75 & 65.93 & 47.30 & 56.86 & $\mathrm{PF}$ & \\
\hline 22. & Yamabiko & 87.92 & 84.78 & 27.17 & 32.97 & PS & \\
\hline 23. & Sweon-287 & 95.79 & 92.37 & 7.25 & 9.32 & $\mathrm{~S}$ & \\
\hline 24. & Hunen Wase & 97.33 & 95.45 & 10.04 & 13.07 & $\mathrm{~S}$ & \\
\hline 25. & Aokaze & 89.52 & 87.09 & 28.38 & 37.82 & PS & \\
\hline 26. & Aki Hi Kari & 88.02 & 86.25 & 28.10 & 36.98 & PS & \\
\hline 27. & Kogahikari & 93.10 & 91.39 & 5.67 & 8.13 & $\mathrm{~S}$ & \\
\hline 28. & Chinese Variety 2 & 95.07 & 92.48 & 8.12 & 9.30 & $\mathrm{~S}$ & \\
\hline 29. & Chinese Variety 5 & 87.45 & 85.30 & 26.87 & 33.89 & PS & \\
\hline 30. & Chinese Variety 8 & 96.82 & 94.50 & 9.05 & 11.27 & $\mathrm{~S}$ & \\
\hline 31. & Purbachi & 100.00 & 100.00 & 2.35 & 4.05 & CS & Maintainer \\
\hline 32. & BR7-51(78)-3 & 49.59 & 44.30 & 38.50 & 46.93 & PF & \\
\hline 33. & BR11-54-1-1 & 82.40 & 78.25 & 22.63 & 27.98 & PS & \\
\hline 34. & BR220-1-1-1 & 59.95 & 55.47 & 43.95 & 51.72 & $\mathrm{PF}$ & \\
\hline 35. & BR707C-MR-137 & 86.42 & 82.30 & 25.07 & 31.13 & PS & \\
\hline 36. & BR7109-5R-2 & 64.97 & 60.10 & 45.63 & 44.65 & $\mathrm{PF}$ & \\
\hline 37. & BR7109-5R-4 & 68.72 & 65.70 & 47.48 & 56.80 & $\mathrm{PF}$ & \\
\hline 38. & BRRI dhan29-SC3-27 & 59.78 & 55.80 & 43.95 & 52.79 & $\mathrm{PF}$ & \\
\hline 39. & IR1544-38-2-2 & 81.20 & 76.35 & 20.87 & 24.95 & PS & \\
\hline 40. & IR11248-148-3-2-33 & 62.73 & 58.42 & 45.43 & 53.75 & PF & \\
\hline 41. & IR19660-73-4-2 & 79.20 & 75.35 & 19.97 & 23.45 & PS & \\
\hline 42. & IR19661-13 & 49.52 & 45.30 & 38.77 & 47.50 & PF & \\
\hline 43. & IR69515-KKN-4-UBN-4-2-1-1 & 27.33 & 24.20 & 58.35 & 67.50 & $\mathrm{~F}$ & \\
\hline 44. & IR72022-462-3-3-2 & 79.73 & 75.47 & 20.09 & 24.73 & PS & \\
\hline 45. & IR72593-B-3-2-2-2 & 29.32 & 26.47 & 60.36 & 68.90 & $\mathrm{~F}$ & \\
\hline 46. & IR74963-262-5-1-3-3 & 86.37 & 83.19 & 25.62 & 31.13 & PS & \\
\hline 47. & IR77034-B-4-3-3 & 59.47 & 55.32 & 43.08 & 51.75 & PF & \\
\hline 48. & IR9447-P1-P2-4B & 69.75 & 67.22 & 48.93 & 57.85 & PF & \\
\hline 49. & IR9708-5-1-2 & 28.30 & 26.27 & 59.63 & 67.50 & $\mathrm{~F}$ & \\
\hline
\end{tabular}

$\mathrm{CS}=$ Completely sterile, $\mathrm{S}=$ Sterile, $\mathrm{PS}=$ Partially sterile, $\mathrm{F}=$ Fertile, and FF = Fully fertile

Identification of Maintainer and Restorer Lines Against D.Shana

Among crosses between D.ShanA and 49 local and exotic rice elite breeding lines the highest (100\%) pollen sterility was observed in D.ShanA $\times$ BAU553, D. ShanA $\times$ Hare Bare, D.ShanA $\times$ Koshinihisini, D.ShanA $\times$ Yamabiko, D.ShanA $\times$ Aokaze, D.ShanA $\times$ Aki Hi Kari, and D.ShanA $\times$ Kogahikari and the lowest was $14.79 \%$ (D.ShanA $\times$ BR368-15-2-2-3) in T. Aman/2009 season. On the other hand, spikelet fertility was the lowest in D.ShanA $\times$ Aki Hi Kari $(1.34 \%)$ and the highest in D.ShanA $\times$ BAU581 (82.50 \%) in T. Aman/2009 season (Table 5). After compilation of the results, it appeared that seven crosses were completely sterile, four crosses sterile, twelve crosses partial sterile, twelve crosses partial fertile, six crosses fertile and eight crosses were fully fertile (Table 8). The pollen parents (BAU553, Hare Bare, Koshinihisini, Yamabiko, Aokaze, Aki Hi Kari, and Kogahikari) of complete sterile crosses might be maintainers against D.ShanA (Table 9) The pollen parent (BR368-15-2-2-3, BR6592-4-6-4, BR6723-1-1-2, BR6839-41-5-1, BR7011-37-1-2, IR9202-36-3-2, IR72049-B-R-22-3-1-1, and IR72593-B-3-2-2-2) of fully fertile cross might be denoted as restorer against D.ShanA (Table 10). 
Table 4 Sterility maintenance and fertility restoration ability of 49 elite lines against alien CMS line, BRRI1A

\begin{tabular}{|c|c|c|c|c|c|c|c|}
\hline \multirow{2}{*}{ Sl. No. } & \multirow{2}{*}{ Pollen parents } & \multicolumn{2}{|c|}{ Pollen sterility $(\%)$ of $\mathrm{F}_{1}$} & \multicolumn{2}{|c|}{ Spikelet fertility $(\%)$ of $\mathrm{F}_{1}$} & \multirow[t]{2}{*}{ Status } & \multirow[t]{2}{*}{ Remarks } \\
\hline & & T.Aman/09 & Boro/09-10 & T.Aman/09 & Boro/09-10 & & \\
\hline 1. & BR368-15-2-2-3 & 69.78 & 65.32 & 47.83 & 56.01 & PF & \\
\hline 2. & BR601-1-3-3-4-2-4 & 81.22 & 78.07 & 20.50 & 27.16 & PS & \\
\hline 3. & BR5563-3-3-4-1 (BRRI dhan48) & 18.38 & 15.73 & 83.82 & 85.50 & FF & Restorer \\
\hline 4. & BR6592-4-6-4 (BRRI dhan49) & 25.03 & 22.49 & 55.27 & 64.45 & $\mathrm{~F}$ & \\
\hline 5. & BR6723-1-1-2 & 96.47 & 93.21 & 8.20 & 10.79 & S & \\
\hline 6. & BR6839-41-5-1 & 26.37 & 22.44 & 56.12 & 64.25 & $\mathrm{~F}$ & \\
\hline 7. & BR7011-37-1-2 & 79.25 & 76.09 & 20.54 & 24.90 & PS & \\
\hline 8. & BAU553 & 100.00 & 100.00 & 2.75 & 4.36 & $\mathrm{CS}$ & Maintainer \\
\hline 9. & BAU572 & 86.20 & 83.82 & 25.70 & 32.19 & PS & \\
\hline 10. & BAU581 & 100.00 & 100.00 & 1.98 & 3.75 & $\mathrm{CS}$ & Maintainer \\
\hline 11. & IR7676-12-1-2 & 78.95 & 75.32 & 19.30 & 23.25 & PS & \\
\hline 12. & IR9202-36-3-2 & 89.21 & 86.07 & 28.45 & 34.19 & PS & \\
\hline 13. & IR25924-51-2-3 & 84.44 & 81.25 & 23.89 & 29.50 & PS & \\
\hline 14. & IR61228-3B-10-3-2 & 47.09 & 44.32 & 37.20 & 47.07 & PF & \\
\hline 15. & IR72049-B-R-22-3-1-1 & 24.49 & 21.25 & 54.29 & 63.54 & $\mathrm{~F}$ & \\
\hline 16. & IR74052-184-3-3 & 39.07 & 35.62 & 33.95 & 42.51 & $\mathrm{PF}$ & \\
\hline 17. & OM1490 & 26.74 & 23.70 & 55.83 & 65.28 & $\mathrm{~F}$ & \\
\hline 18. & BKNLR-75091-GNT-B3-RST-48-2-2 & 44.92 & 40.07 & 37.21 & 45.20 & PF & \\
\hline 19. & Moroberekan & 30.00 & 27.32 & 61.12 & 71.75 & $\mathrm{~F}$ & \\
\hline 20. & Hare Bare & 95.60 & 91.30 & 8.03 & 10.13 & S & \\
\hline 21. & Koshinihisini & 98.25 & 94.68 & 10.41 & 12.39 & S & \\
\hline 22. & Yamabiko & 97.72 & 93.57 & 9.10 & 11.50 & $\mathrm{~S}$ & \\
\hline 23. & Sweon-287 & 100.00 & 100.00 & 1.39 & 3.78 & CS & Maintainer \\
\hline 24. & Hunen Wase & 75.38 & 72.85 & 16.25 & 20.63 & PS & \\
\hline 25. & Aokaze & 100.00 & 100.00 & 2.75 & 4.56 & CS & Maintainer \\
\hline 26. & Aki Hi Kari & 100.00 & 100.00 & 1.95 & 4.27 & CS & Maintainer \\
\hline 27. & Kogahikari & 100.00 & 100.00 & 2.10 & 3.85 & CS & Maintainer \\
\hline 28. & Chinese Variety 2 & 86.20 & 83.82 & 25.70 & 32.19 & PS & \\
\hline 29. & Chinese Variety 5 & 94.79 & 91.30 & 8.35 & 10.12 & $\mathrm{~S}$ & \\
\hline 30. & Chinese Variety 8 & 87.99 & 85.35 & 27.10 & 38.25 & PS & \\
\hline 31. & Purbachi & 100.00 & 100.00 & 1.33 & 3.57 & $\mathrm{CS}$ & Maintainer \\
\hline 32. & BR7-51(78)-3 & 41.53 & 38.93 & 34.75 & 43.79 & PF & \\
\hline 33. & BR11-54-1-1 & 49.37 & 45.04 & 39.50 & 47.30 & $\mathrm{PF}$ & \\
\hline 34. & BR220-1-1-1 & 56.79 & 53.07 & 41.87 & 51.20 & $\mathrm{PF}$ & \\
\hline 35. & BR707C-MR-137 & 79.27 & 76.23 & 19.72 & 24.25 & PS & \\
\hline 36. & BR7109-5R-2 & 86.98 & 83.07 & 25.75 & 31.82 & PS & \\
\hline 37. & BR7109-5R-4 & 47.92 & 44.63 & 37.26 & 46.50 & $\mathrm{PF}$ & \\
\hline 38. & BRRI dhan29-SC3-27 & 56.87 & 52.29 & 41.87 & 52.10 & $\mathrm{PF}$ & \\
\hline 39. & IR1544-38-2-2 & 80.09 & 76.31 & 20.65 & 24.09 & PS & \\
\hline 40. & IR11248-148-3-2-33 & 81.26 & 77.25 & 21.37 & 25.25 & PS & \\
\hline 41. & IR19660-73-4-2 & 26.79 & 23.21 & 56.21 & 64.23 & $\mathrm{~F}$ & \\
\hline 42. & IR19661-13 & 68.42 & 65.35 & 47.08 & 56.10 & $\mathrm{PF}$ & \\
\hline 43. & IR69515-KKN-4-UBN-4-2-1-1 & 61.73 & 58.21 & 45.01 & 53.27 & PF & \\
\hline 44. & IR72022-462-3-3-2 & 26.25 & 23.47 & 55.92 & 64.25 & $\mathrm{~F}$ & \\
\hline 45. & IR72593-B-3-2-2-2 & 14.56 & 11.98 & 82.75 & 84.39 & $\mathrm{FF}$ & Restorer \\
\hline 46. & IR74963-262-5-1-3-3 & 41.97 & 38.21 & 35.74 & 44.13 & PF & \\
\hline 47. & IR77034-B-4-3-3 & 82.46 & 79.47 & 21.80 & 27.58 & PS & \\
\hline 48. & IR9447-P1-P2-4B & 65.96 & 62.10 & 46.63 & 56.10 & PF & \\
\hline 49. & IR9708-5-1-2 & 15.79 & 13.75 & 80.75 & 82.50 & FF & Restorer \\
\hline
\end{tabular}

CS = Completely sterile, $\mathrm{S}=$ Sterile, PS = Partially sterile, F = Fertile, and FF = Fully fertile

Forty three potential maintainers and 10 effective restorers were identified from 264 testcross $\mathrm{F}_{1} \mathrm{~S}$ using 11 CMS lines including D.ShanA, IR58025A, BRRI1A and BRRI9A during T. Aman, 2007 (Anon, 2010). Again, 2 potential maintainers and 4 restorers were identified from 213 testcrosses using 59 CMS lines including D.ShanA, IR73328A, BRRI1A and BRRI9A during Boro/2007-08 (Anon, 2010). Jayamani et al. (1998) found $F_{1}$ of V20A $\times$ Dular as completely sterile and used for subsequent backcrossing up to $\mathrm{BC}_{6}$ generation. Singh (2005) identified 41 potential restorers and 11 effective maintainers from $75 \mathrm{~F}_{1} \mathrm{~s}$ against different CMS lines.
Identification of Maintainer and Restorer Lines Against BRRI9A

Among crosses between BRRI9A and 49 rice elite breeding lines, the highest $(100 \%)$ pollen sterility was observed in BRRI9A $\times$ BAU581, BRRI9A $\times$ Chinese Variety 5, and BRRI9A $\times$ Purbachi and the lowest was $15.47 \%$ (BRRI9A $\times$ IR25924-51-2-3) in T. Aman/2009 season. On the other hand, spikelet fertility was the lowest in BRRI9A $\times$ BAU581 $(1.35 \%)$ and the highest in BRRI9A $\times$ Moroberekan $(83.80 \%)$ in T. Aman/2009 season (Table 6). 
Table 5 Sterility maintenance and fertility restoration ability of 49 elite lines against alien CMS line, D.ShanA

\begin{tabular}{|c|c|c|c|c|c|c|c|}
\hline \multirow{2}{*}{ S1. No. } & \multirow{2}{*}{ Pollen parents } & \multicolumn{2}{|c|}{ Pollen sterility $(\%)$ of $\mathrm{F}_{1}$} & \multicolumn{2}{|c|}{ Spikelet fertility $(\%)$ of $\mathrm{F}_{1}$} & \multirow[t]{2}{*}{ Status } & \multirow[t]{2}{*}{ Remarks } \\
\hline & & T.Aman/09 & Boro/09-10 & T.Aman/09 & Boro/09-10 & & \\
\hline 1. & BR368-15-2-2-3 & 14.79 & 12.78 & 80.25 & 83.31 & FF & Restorer \\
\hline 2. & BR601-1-3-3-4-2-4 & 58.74 & 55.32 & 42.97 & 52.79 & $\mathrm{PF}$ & \\
\hline 3. & BR5563-3-3-4-1 (BRRI dhan48) & 61.23 & 67.09 & 45.02 & 58.20 & $\mathrm{PF}$ & \\
\hline 4. & BR6592-4-6-4 (BRRI dhan49) & 16.03 & 14.09 & 80.35 & 83.59 & $\mathrm{FF}$ & Restorer \\
\hline 5. & BR6723-1-1-2 & 19.50 & 16.78 & 81.14 & 84.71 & $\mathrm{FF}$ & Restorer \\
\hline 6. & BR6839-41-5-1 & 17.35 & 15.09 & 80.05 & 83.98 & FF & Restorer \\
\hline 7. & BR7011-37-1-2 & 19.03 & 17.03 & 81.90 & 85.40 & FF & Restorer \\
\hline 8. & BAU553 & 100.00 & 100.00 & 1.49 & 3.02 & $\mathrm{CS}$ & Maintainer \\
\hline 9. & BAU572 & 88.95 & 86.20 & 27.34 & 34.75 & PS & \\
\hline 10. & BAU581 & 87.95 & 84.52 & 27.20 & 32.20 & PS & \\
\hline 11. & IR7676-12-1-2 & 69.75 & 65.20 & 48.71 & 57.50 & $\mathrm{PF}$ & \\
\hline 12. & IR9202-36-3-2 & 17.39 & 15.37 & 80.80 & 83.74 & $\mathrm{FF}$ & Restorer \\
\hline 13. & IR25924-51-2-3 & 25.09 & 22.76 & 55.67 & 64.25 & $\mathrm{~F}$ & \\
\hline 14. & IR61228-3B-10-3-2 & 29.53 & 27.20 & 60.14 & 70.75 & $\mathrm{~F}$ & \\
\hline 15. & IR72049-B-R-22-3-1-1 & 16.07 & 13.45 & 80.08 & 83.25 & $\mathrm{FF}$ & Restorer \\
\hline 16. & IR74052-184-3-3 & 27.33 & 25.37 & 58.10 & 67.50 & $\mathrm{~F}$ & \\
\hline 17. & OM1490 & 49.47 & 44.35 & 38.17 & 46.06 & $\mathrm{PF}$ & \\
\hline 18. & BKNLR-75091-GNT-B3-RST-48-2-2 & 28.74 & 26.30 & 28.10 & 68.93 & $\mathrm{~F}$ & \\
\hline 19. & Moroberekan & 29.32 & 24.75 & 29.57 & 66.24 & $\mathrm{~F}$ & \\
\hline 20. & Hare Bare & 100.00 & 100.00 & 1.37 & 2.95 & CS & Maintainer \\
\hline 21. & Koshinihisini & 100.00 & 100.00 & 2.09 & 4.67 & CS & Maintainer \\
\hline 22. & Yamabiko & 100.00 & 100.00 & 1.98 & 3.49 & CS & Maintainer \\
\hline 23. & Sweon-287 & 97.43 & 95.83 & 10.10 & 12.70 & $\mathrm{~S}$ & \\
\hline 24. & Hunen Wase & 88.95 & 86.20 & 27.34 & 34.75 & PS & \\
\hline 25. & Aokaze & 100.00 & 100.00 & 2.47 & 5.09 & CS & Maintainer \\
\hline 26. & Aki Hi Kari & 100.00 & 100.00 & 1.34 & 3.29 & CS & Maintainer \\
\hline 27. & Kogahikari & 100.00 & 100.00 & 2.33 & 4.87 & CS & Maintainer \\
\hline 28. & Chinese Variety 2 & 87.09 & 85.38 & 27.26 & 33.80 & PS & \\
\hline 29. & Chinese Variety 5 & 94.30 & 92.45 & 6.78 & 9.30 & $\mathrm{~S}$ & \\
\hline 30. & Chinese Variety 8 & 95.92 & 94.38 & 7.25 & 10.10 & S & \\
\hline 31. & Purbachi & 98.75 & 96.87 & 10.26 & 13.89 & $\mathrm{~S}$ & \\
\hline 32. & BR7-51(78)-3 & 76.78 & 72.30 & 17.97 & 20.63 & PS & \\
\hline 33. & BR11-54-1-1 & 62.09 & 56.70 & 45.30 & 53.50 & $\mathrm{PF}$ & \\
\hline 34. & BR220-1-1-1 & 53.23 & 48.20 & 41.71 & 48.20 & $\mathrm{PF}$ & \\
\hline 35. & BR707C-MR-137 & 86.27 & 82.49 & 27.03 & 32.37 & PS & \\
\hline 36. & BR7109-5R-2 & 61.20 & 58.30 & 44.95 & 53.20 & $\mathrm{PF}$ & \\
\hline 37. & BR7109-5R-4 & 86.27 & 83.92 & 25.87 & 32.17 & PS & \\
\hline 38. & BRRI dhan29-SC3-27 & 54.82 & 50.37 & 40.87 & 50.12 & $\mathrm{PF}$ & \\
\hline 39. & IR1544-38-2-2 & 87.95 & 84.52 & 27.20 & 32.20 & PS & \\
\hline 40. & IR11248-148-3-2-33 & 81.76 & 78.22 & 21.50 & 27.15 & PS & \\
\hline 41. & IR19660-73-4-2 & 60.07 & 56.30 & 44.35 & 53.25 & $\mathrm{PF}$ & \\
\hline 42. & IR19661-13 & 24.95 & 21.27 & 55.60 & 63.89 & $\mathrm{~F}$ & \\
\hline 43. & IR69515-KKN-4-UBN-4-2-1-1 & 79.97 & 75.60 & 19.80 & 23.25 & PS & \\
\hline 44. & IR72022-462-3-3-2 & 49.49 & 45.30 & 39.05 & 47.50 & PF & \\
\hline 45. & IR72593-B-3-2-2-2 & 18.47 & 15.03 & 81.26 & 84.50 & $\mathrm{FF}$ & Restorer \\
\hline 46. & IR74963-262-5-1-3-3 & 69.03 & 66.20 & 48.20 & 58.10 & $\mathrm{PF}$ & \\
\hline 47. & IR77034-B-4-3-3 & 81.23 & 76.95 & 21.09 & 24.58 & PS & \\
\hline 48. & IR9447-P1-P2-4B & 85.69 & 82.30 & 24.93 & 30.10 & PS & \\
\hline 49. & IR9708-5-1-2 & 47.93 & 43.78 & 38.85 & 46.05 & PF & \\
\hline
\end{tabular}

CS = Completely sterile, $\mathrm{S}=$ Sterile, $\mathrm{PS}=$ Partially sterile, F = Fertile, and FF = Fully fertile

After compilation of the results, it appeared that three crosses were completely sterile, seven crosses sterile, nine crosses partial sterile, nineteen crosses partial fertile, six crosses fertile and five crosses were fully fertile (Table 8). The pollen parents (BAU581, Chinese Variety 5 and Purbachi) of complete sterile crosses might be maintainers against BRRI9A (Table 9). The pollen parent (IR25924-51-2-3, IR72049-B-R-22-3-1-1, IR74052-1843-3, OM1490, and Moroberekan) of fully fertile cross might be denoted as restorer against BRRI9A (Table 10). Forty three potential maintainers and 10 effective restorers were identified from 264 testcross $F_{1}$ s using 11
CMS lines including D.ShanA, IR58025A, BRRI1A, and BRRI9A during T. Aman/2007 (Anon, 2010). Again, 2 potential maintainers and 4 restorers were identified from 213 testcrosses using 59 CMS lines including D.ShanA, IR73328A, BRRI1A, and BRRI9A during Boro/2007-08 (Anon., 2010). Jayamani et al. (1998) found $F_{1}$ of V20A $\times$ Dular as completely sterile and used for subsequent backcrossing up to $\mathrm{BC}_{6}$ generation. Umadevi et al. (2010) identified 10 restorers from 248 testcross hybrids made by crossing 8 CMS lines with 31 genotypes. 
Table 6 Sterility maintenance and fertility restoration ability of 49 elite lines against alien CMS line, BRRI9A

\begin{tabular}{|c|c|c|c|c|c|c|c|}
\hline \multirow{2}{*}{ S1. No. } & \multirow{2}{*}{ Pollen parents } & \multicolumn{2}{|c|}{ Pollen sterility $(\%)$ of $\mathrm{F}_{1}$} & \multicolumn{2}{|c|}{ Spikelet fertility $(\%)$ of $\mathrm{F}_{1}$} & \multirow[t]{2}{*}{ Status } & \multirow[t]{2}{*}{ Remarks } \\
\hline & & T.Aman/09 & Boro/09-10 & T.man/09 & Boro/09-10 & & \\
\hline 1. & BR368-15-2-2-3 & 49.09 & 44.75 & 39.72 & 50.60 & $\mathrm{PF}$ & \\
\hline 2. & BR601-1-3-3-4-2-4 & 79.22 & 75.37 & 21.93 & 28.78 & PS & \\
\hline 3. & BR5563-3-3-4-1 (BRRI dhan48) & 96.57 & 93.49 & 8.56 & 12.03 & $\mathrm{~S}$ & \\
\hline 4. & BR6592-4-6-4 (BRRI dhan49) & 25.39 & 22.57 & 54.74 & 66.07 & $\mathrm{~F}$ & \\
\hline 5. & BR6723-1-1-2 & 96.45 & 93.21 & 7.98 & 10.54 & $\mathrm{~S}$ & \\
\hline 6. & BR6839-41-5-1 & 49.30 & 45.92 & 40.17 & 49.35 & $\mathrm{PF}$ & \\
\hline 7. & BR7011-37-1-2 & 59.27 & 55.03 & 43.28 & 52.87 & $\mathrm{PF}$ & \\
\hline 8. & BAU553 & 65.79 & 62.13 & 46.39 & 57.23 & $\mathrm{PF}$ & \\
\hline 9. & BAU572 & 68.90 & 63.80 & 48.08 & 58.07 & $\mathrm{PF}$ & \\
\hline 10. & BAU581 & 100.00 & 100.00 & 1.35 & 2.89 & CS & Maintainer \\
\hline 11. & IR7676-12-1-2 & 49.07 & 44.79 & 40.30 & 47.93 & PF & \\
\hline 12. & IR9202-36-3-2 & 64.09 & 61.20 & 46.74 & 53.89 & PF & \\
\hline 13. & IR25924-51-2-3 & 15.47 & 12.38 & 80.65 & 83.78 & FF & Restorer \\
\hline 14. & IR61228-3B-10-3-2 & 59.61 & 55.30 & 44.31 & 52.39 & PF & \\
\hline 15. & IR72049-B-R-22-3-1-1 & 16.89 & 13.72 & 81.07 & 84.75 & $\mathrm{FF}$ & Restorer \\
\hline 16. & IR74052-184-3-3 & 17.76 & 15.85 & 82.10 & 85.87 & FF & Restorer \\
\hline 17. & OM1490 & 16.75 & 14.78 & 82.85 & 85.02 & FF & Restorer \\
\hline 18. & BKNLR-75091-GNT-B3-RST-48-2-2 & 26.09 & 23.18 & 56.25 & 66.74 & $\mathrm{~F}$ & \\
\hline 19. & Moroberekan & 18.25 & 16.09 & 83.80 & 86.20 & $\mathrm{FF}$ & Restorer \\
\hline 20. & Hare Bare & 89.75 & 87.73 & 28.50 & 36.51 & PS & \\
\hline 21. & Koshinihisini & 95.86 & 93.38 & 7.96 & 12.69 & $\mathrm{~S}$ & \\
\hline 22. & Yamabiko & 94.52 & 91.26 & 6.72 & 10.83 & $\mathrm{~S}$ & \\
\hline 23. & Sweon-287 & 87.58 & 85.47 & 26.85 & 34.62 & PS & \\
\hline 24. & Hunen Wase & 86.92 & 84.35 & 25.10 & 33.07 & PS & \\
\hline 25. & Aokaze & 95.03 & 92.97 & 7.89 & 11.52 & $\mathrm{~S}$ & \\
\hline 26. & Aki Hi Kari & 96.45 & 93.87 & 8.12 & 12.07 & S & \\
\hline 27. & Kogahikari & 95.30 & 91.42 & 7.82 & 11.51 & $\mathrm{~S}$ & \\
\hline 28. & Chinese Variety 2 & 68.24 & 65.15 & 47.20 & 56.39 & $\mathrm{PF}$ & \\
\hline 29. & Chinese Variety 5 & 100.00 & 100.00 & 2.15 & 4.02 & $\mathrm{CS}$ & Maintainer \\
\hline 30. & Chinese Variety 8 & 89.20 & 85.92 & 27.92 & 32.10 & PS & \\
\hline 31. & Purbachi & 100.00 & 100.00 & 1.97 & 3.87 & $\mathrm{CS}$ & Maintainer \\
\hline 32. & BR7-51(78)-3 & 49.23 & 45.60 & 40.12 & 48.59 & PF & \\
\hline 33. & BR11-54-1-1 & 59.07 & 56.21 & 42.67 & 51.33 & $\mathrm{PF}$ & \\
\hline 34. & BR220-1-1-1 & 65.34 & 62.13 & 45.89 & 55.10 & $\mathrm{PF}$ & \\
\hline 35. & BR707C-MR-137 & 62.44 & 59.25 & 43.10 & 53.79 & PF & \\
\hline 36. & BR7109-5R-2 & 69.35 & 67.22 & 48.30 & 58.18 & $\mathrm{PF}$ & \\
\hline 37. & BR7109-5R-4 & 39.74 & 35.24 & 34.75 & 42.27 & $\mathrm{PF}$ & \\
\hline 38. & BRRI dhan29-SC3-27 & 29.37 & 25.66 & 60.29 & 67.50 & $\mathrm{~F}$ & \\
\hline 39. & IR1544-38-2-2 & 77.49 & 73.20 & 18.72 & 22.85 & PS & \\
\hline 40. & IR11248-148-3-2-33 & 89.94 & 85.74 & 28.79 & 33.75 & PS & \\
\hline 41. & IR19660-73-4-2 & 76.22 & 73.21 & 17.25 & 23.25 & PS & \\
\hline 42. & IR19661-13 & 58.47 & 55.30 & 43.03 & 52.97 & PF & \\
\hline 43. & IR69515-KKN-4-UBN-4-2-1-1 & 70.00 & 68.10 & 49.01 & 58.23 & PF & \\
\hline 44. & IR72022-462-3-3-2 & 27.95 & 25.67 & 57.45 & 67.50 & $\mathrm{~F}$ & \\
\hline 45. & IR72593-B-3-2-2-2 & 25.07 & 21.45 & 55.87 & 63.28 & $\mathrm{~F}$ & \\
\hline 46. & IR74963-262-5-1-3-3 & 76.75 & 73.21 & 16.25 & 23.76 & PS & \\
\hline 47. & IR77034-B-4-3-3 & 66.27 & 63.97 & 45.67 & 56.30 & PF & \\
\hline 48. & IR9447-P1-P2-4B & 27.10 & 23.09 & 58.25 & 65.89 & $\mathrm{~F}$ & \\
\hline 49. & IR9708-5-1-2 & 39.57 & 35.30 & 34.73 & 43.70 & PF & \\
\hline
\end{tabular}

CS = Completely sterile, $\mathrm{S}=$ Sterile, $\mathrm{PS}=$ Partially sterile, F = Fertile, and FF = Fully fertile

Identification of Maintainer and Restorer Lines
Against IR73328A
Among crosses between IR73328A and 49 local and exotic rice elite breeding lines, the highest $(100 \%)$ pollen sterility was observed in IR73328A $\times$ BAU553, IR73328A $\times$ BAU572, IR73328A $\times$ BAU581, and IR73328A $\times$ Chinese Variety 5 and the lowest was $19.72 \%($ IR73328A $\times$ BR7011-37-1-2) in T. Aman/2009 season. On the other hand, spikelet fertility was the lowest in IR73328A $\times$ BAU572 $(1.53 \%)$ and the highest in IR73328A $\times$ BR7011-37-1-2 (80.92\%) in T. Aman/2009 season (Table 7). After compilation of the results, it appeared that four crosses were completely sterile, six crosses sterile, eleven crosses partial sterile, seventeen crosses partial fertile, ten crosses fertile and a single cross was fully fertile (Table 8). The pollen parents (BAU553, BAU572, BAU581, and Chinese Variety 5) of complete sterile crosses might be maintainers against IR73328A (Table 9). The pollen parent (BR7011-37-1-2) of fully fertile cross might be denoted as restorer against IR73328A (Table 10). Two potential maintainers and 4 restorers were identified from 213 testcrosses using 59 CMS lines including D.ShanA, IR73328A, BRRI1A, and BRRI9A during Boro, 2007-08 (Anon, 2010). 
Table 7 Sterility maintenance and fertility restoration ability of 49 elite lines against alien CMS line, IR73328A

\begin{tabular}{|c|c|c|c|c|c|c|c|}
\hline \multirow{2}{*}{ Sl. No. } & \multirow{2}{*}{ Pollen parents } & \multicolumn{2}{|c|}{ Pollen sterility $(\%)$ of $\mathrm{F}_{1}$} & \multicolumn{2}{|c|}{ Spikelet fertility $(\%)$ of $\mathrm{F}_{1}$} & \multirow[t]{2}{*}{ Status } & \multirow[t]{2}{*}{ Remarks } \\
\hline & & T.Aman/09 & Boro/09-10 & T.Aman/09 & Boro/09-10 & & \\
\hline 1. & BR368-15-2-2-3 & 24.75 & 21.30 & 50.12 & 65.53 & $\mathrm{~F}$ & \\
\hline 2. & BR601-1-3-3-4-2-4 & 59.34 & 55.60 & 32.95 & 52.78 & $\mathrm{PF}$ & \\
\hline 3. & BR5563-3-3-4-1 (BRRI dhan48) & 28.90 & 26.70 & 60.12 & 73.50 & $\mathrm{~F}$ & \\
\hline 4. & BR6592-4-6-4 (BRRI dhan49) & 65.09 & 62.10 & 33.87 & 54.17 & $\mathrm{PF}$ & \\
\hline 5. & BR6723-1-1-2 & 69.95 & 67.10 & 40.12 & 60.21 & $\mathrm{PF}$ & \\
\hline 6. & BR6839-41-5-1 & 59.07 & 54.20 & 39.71 & 55.08 & $\mathrm{PF}$ & \\
\hline 7. & BR7011-37-1-2 & 19.72 & 17.39 & 80.92 & 84.20 & $\mathrm{FF}$ & Restorer \\
\hline 8. & BAU553 & 100.00 & 100.00 & 1.74 & 3.45 & $\mathrm{CS}$ & Maintainer \\
\hline 9. & BAU572 & 100.00 & 100.00 & 1.53 & 4.75 & $\mathrm{CS}$ & Maintainer \\
\hline 10. & BAU581 & 100.00 & 100.00 & 2.17 & 4.32 & $\mathrm{CS}$ & Maintainer \\
\hline 11. & IR7676-12-1-2 & 60.10 & 56.70 & 46.78 & 55.20 & $\mathrm{PF}$ & \\
\hline 12. & IR9202-36-3-2 & 25.07 & 22.30 & 57.12 & 71.85 & $\mathrm{~F}$ & \\
\hline 13. & IR25924-51-2-3 & 29.30 & 25.65 & 60.98 & 73.12 & $\mathrm{~F}$ & \\
\hline 14. & IR61228-3B-10-3-2 & 80.30 & 75.95 & 24.57 & 30.13 & PS & \\
\hline 15. & IR72049-B-R-22-3-1-1 & 29.75 & 27.10 & 61.00 & 73.10 & $\mathrm{~F}$ & \\
\hline 16. & IR74052-184-3-3 & 27.10 & 23.25 & 58.29 & 70.52 & $\mathrm{~F}$ & \\
\hline 17. & OM1490 & 23.79 & 21.15 & 55.67 & 67.32 & $\mathrm{~F}$ & \\
\hline 18. & BKNLR-75091-GNT-B3-RST-48-2-2 & 50.10 & 46.20 & 41.98 & 50.91 & $\mathrm{PF}$ & \\
\hline 19. & Moroberekan & 26.07 & 23.10 & 55.32 & 65.78 & $\mathrm{~F}$ & \\
\hline 20. & Hare Bare & 78.87 & 75.83 & 20.19 & 29.38 & PS & \\
\hline 21. & Koshinihisini & 96.89 & 92.09 & 8.53 & 15.45 & $\mathrm{~S}$ & \\
\hline 22. & Yamabiko & 87.08 & 83.25 & 23.74 & 32.97 & PS & \\
\hline 23. & Sweon-287 & 95.42 & 92.07 & 7.97 & 14.92 & $\mathrm{~S}$ & \\
\hline 24. & Hunen Wase & 81.38 & 78.26 & 21.08 & 32.97 & PS & \\
\hline 25. & Aokaze & 97.96 & 93.67 & 9.12 & 14.25 & $\mathrm{~S}$ & \\
\hline 26. & Aki Hi Kari & 88.90 & 86.23 & 27.12 & 36.57 & PS & \\
\hline 27. & Kogahikari & 94.70 & 92.35 & 7.95 & 12.57 & $\mathrm{~S}$ & \\
\hline 28. & Chinese Variety 2 & 86.79 & 83.40 & 25.32 & 33.12 & PS & \\
\hline 29. & Chinese Variety 5 & 100.00 & 100.00 & 1.57 & 2.95 & $\mathrm{CS}$ & Maintainer \\
\hline 30. & Chinese Variety 8 & 94.57 & 91.23 & 7.89 & 12.12 & $\mathrm{~S}$ & \\
\hline 31. & Purbachi & 96.75 & 93.60 & 6.78 & 10.34 & $\mathrm{~S}$ & \\
\hline 32. & BR7-51(78)-3 & 59.47 & 55.30 & 43.17 & 51.20 & $\mathrm{PF}$ & \\
\hline 33. & BR11-54-1-1 & 83.75 & 80.04 & 19.92 & 31.12 & PS & \\
\hline 34. & BR220-1-1-1 & 67.92 & 64.25 & 46.30 & 55.65 & $\mathrm{PF}$ & \\
\hline 35. & BR707C-MR-137 & 56.00 & 53.21 & 43.07 & 51.21 & $\mathrm{PF}$ & \\
\hline 36. & BR7109-5R-2 & 79.27 & 76.92 & 20.10 & 31.74 & PS & \\
\hline 37. & BR7109-5R-4 & 66.92 & 63.10 & 43.79 & 54.92 & $\mathrm{PF}$ & \\
\hline 38. & BRRI dhan29-SC3-27 & 28.10 & 25.60 & 59.82 & 73.12 & $\mathrm{~F}$ & \\
\hline 39. & IR1544-38-2-2 & 47.07 & 43.20 & 36.97 & 50.39 & PF & \\
\hline 40. & IR11248-148-3-2-33 & 86.92 & 83.10 & 26.81 & 36.73 & PS & \\
\hline 41. & IR19660-73-4-2 & 76.22 & 72.97 & 19.12 & 23.04 & PS & \\
\hline 42. & IR19661-13 & 61.95 & 58.20 & 46.07 & 53.17 & $\mathrm{PF}$ & \\
\hline 43. & IR69515-KKN-4-UBN-4-2-1-1 & 53.15 & 50.30 & 42.39 & 50.28 & $\mathrm{PF}$ & \\
\hline 44. & IR72022-462-3-3-2 & 28.10 & 26.30 & 59.10 & 72.87 & $\mathrm{~F}$ & \\
\hline 45. & IR72593-B-3-2-2-2 & 39.49 & 36.10 & 34.82 & 43.85 & $\mathrm{PF}$ & \\
\hline 46. & IR74963-262-5-1-3-3 & 82.37 & 79.24 & 21.95 & 29.34 & PS & \\
\hline 47. & IR77034-B-4-3-3 & 49.05 & 45.27 & 40.12 & 49.17 & PF & \\
\hline 48. & IR9447-P1-P2-4B & 62.79 & 58.20 & 46.87 & 51.98 & $\mathrm{PF}$ & \\
\hline 49. & IR9708-5-1-2 & 65.10 & 60.21 & 47.39 & 54.32 & $\mathrm{PF}$ & \\
\hline
\end{tabular}

$\mathrm{CS}=$ Completely sterile, $\mathrm{S}=$ Sterile, $\mathrm{PS}=$ Partially sterile, $\mathrm{F}=$ Fertile, and FF = Fully fertile

Table 8 Pollen sterility or fertility status of F1s produced by five CMS lines against 49 local and exotic rice elite breeding lines

\begin{tabular}{l|llllll}
\hline \multirow{2}{*}{ CMS line } & \multicolumn{5}{|c}{ No. of pollen parents accounts for } \\
\cline { 2 - 6 } & \multicolumn{1}{c|}{ Completely sterile } & Sterile & Partially sterile & Partially fertile & Fertile & Fully fertile \\
\hline IR58025A & $3(6.12)^{*}$ & $8(16.33)$ & $13(26.53)$ & $14(28.57)$ & $7(14.29)$ & $4(8.16)$ \\
BRRIA & $7(14.29)$ & $5(10.20)$ & $14(28.57)$ & $13(26.53)$ & $7(14.29)$ & $3(6.12)$ \\
D.ShanA & $7(14.29)$ & $4(8.16)$ & $12(24.49)$ & $12(24.49)$ & $6(12.24)$ & $8(16.33)$ \\
BRRI9A & $3(6.12)$ & $7(14.29)$ & $9(18.37)$ & $19(38.78)$ & $6(12.24)$ & $5(10.20)$ \\
IR73328A & $4(8.16)$ & $6(12.24)$ & $11(22.45)$ & $17(34.69)$ & $10(20.41)$ & $1(2.04)$ \\
\hline
\end{tabular}

*Figure in parentheses expressing percentage of total genotypes 
Table 9 Identified maintainers of five alien CMS lines among 49 local and exotic rice elite breeding lines

\begin{tabular}{l|l}
\hline \multicolumn{1}{c|}{ CMS lines } & \multicolumn{1}{c}{ Genotypes identified as maintainers } \\
\hline IR58025A & BAU572, BAU581 and Purbachi \\
BRRI1A & BAU553, BAU581, SWEON-287, AOKAZE, AKI HI KARI, KOGAHIKARI and Purbachi \\
D.ShanA & BAU553, HARE BARE, KOSHINIHISINI, YAMABIKO, AOKAZE, AKI HI KARI and \\
BRRI9A & KOGAHIKARI \\
IR73328A & BAU581, Chinese Variety 5 and Purbachi \\
\hline
\end{tabular}

Table 10 Identified restorers of five alien CMS lines among 49 local and exotic rice elite breeding lines

\begin{tabular}{l|l}
\hline \multicolumn{1}{c|}{ CMS lines } & \multicolumn{1}{c}{ Genotypes identified as restorers } \\
\hline IR58025A & BR6592-4-6-4, BR6839-41-5-1, IR74052-184-3-3 and BKNLR-75091-GNT-B3-RST-48-2-2 \\
BRRI1A & BR5563-3-3-4-1, IR72593-B-3-2-2-2 and IR9708-5-1-2 \\
D.ShanA & BR368-15-2-2-3, BR6592-4-6-4, BR6723-1-1-2, BR6839-41-5-1, BR7011-37-1-2, IR9202-36-3-2, \\
BRRI9A & IR72049-B-R-22-3-1-1 and IR72593-B-3-2-2-2 \\
IR73328A & IR25924-51-2-3, IR72049-B-R-22-3-1-1, IR74052-184-3-3, OM1490 and MOROBEREKAN \\
\hline
\end{tabular}

Jayamani et al. (1998) found $\mathrm{F}_{1}$ of V20A $\times$ Dular as completely sterile and used for subsequent backcrossing up to $\mathrm{BC}_{6}$ generation. Considering pollen sterility and spikelet fertility pattern of the crosses, it appeared that Boro season possessed lower pollen sterility and higher spikelet fertility than those of T. Aman season. Out of forty nine local and exotic rice elite breeding lines, three were identified as maintainers for IR58025A, seven for BRRI1A, seven for D.ShanA, three for BRRI9A and four for IR73328A (Table 9). Among the maintainers, eight genotypes (BAU553, BAU572, BAU581, Purbachi, Aokaze, Aki Hi Kari, Kogahikari, and Chinese Variety 5) were common for these five CMS lines. Ultimately, 12 genotypes were found as maintainer against five CMS lines. The identified complete sterile crosses could be transferred as new CMS line through substitution backcrossing with their respective pollen parents as recurrent parent. Ali and Khan (1995) found 47456 and PK4112 as potential maintainer whereas, 4029-2 and 4029-3 as effective restorers for all the three CMS lines of IR58025A, IR62829A, and IR64608A in 15 non-aromatic rice varieties. Among 49 local and exotic rice elite breeding lines, four were found as restorers for IR58025A, three for BRRI1A, eight for D.ShanA, five for BRRI9A, and one for IR73328A (Table 10). Among the restorers, six genotypes (BR6592-4-6-4, BR6839-41-5-1, IR74052-184-3-3, IR72593-B-3-2-2-2, BR7011-37-1-2, and IR72049-B-R-22-3-1-1) were common for five CMS lines. So, actually 15 genotypes were identified as restorers against five CMS lines. In case of maintainer and restorer identification, same picture appeared that number of maintainers and restorers against D.ShanA was higher than the rest of the four alien CMS lines. Out of 49 genotypes 12 genotypes $(24 \%)$ were identified as maintainers and $15(31 \%)$ were identified as restorers. The findings of this investigation were somewhat different from the results of GobindaRaj and Virmani (1989) who found $40 \%$ maintainer and $24 \%$ restorer when tested 37 indica, japonica and indica/japonica derivatives against CMS-GA. This difference might be due to genetic background of the test genotypes. Jayasudha and Sharma (2010) identified 10 potential restorers based on pollen and spikelet fertility percent. Out of 245 crosses only 45 crosses contributed directly to identify maintainer and restorer. Other crosses were more or, less of intermediate types which indicated neither maintainer nor restorer. It is well known that pollen fertility is controlled by one dominant gene $(R f R f)$. If pollen fertility is governed by only one gene the product would be either restorer or, maintainer, no existence of intermediate types. So, there might be modifier genes in different genotypes which interacted with male sterile nuclear genes and resulted intermediate male sterility in the crosses. Ganesan and Rangaswamy (1998) concluded that minor genes present in the pollinator might have reacted with CMS lines used and resulted in this type of variation. On the contrary, GobindaRaj and Virmani (1988) reported that restoration is governed by two genes. Epistasis or, epistasis with incomplete dominance plays a vital role for restoration. Teng and Shen (1994) also supported the opinions of GobindaRaj and Virmani (1988). Kumari et al. (1998) suggested that the presence of multiple nuclear genes (minor) for fertility in CMS lines whereas, Ali and Khan (1995) concluded that expression of restorer genes varied with the genetic background of female parent. To overcome this problem Singh et al. (2000) proposed that incomplete restoration might be converted to complete restorer or maintainer by special breeding programme.

\section{Conclusion}

Six tester's viz., BR6592-4-6-4, BR6839-41-5-1, IR74052-184-3-3, IR72593-B-3-2-2-2, BR7011-37-1-2, and IR72049-B-R-22-3-1-1 were identified as restorers for highest 2 lines of the five CMS lines, differently. None of them were found to be restorer for all the five CMS lines. BAU581 was found to be maintainer for four lines except for D.ShanA and Purbachi was found to be maintainer for three lines except for D.ShanA and IR73328A out of the five CMS lines. Out of 245 crosses 200 crosses were intermediate type and only 45 crosses contributed directly to identify maintainer and restorer.

\section{References}

Ali NG, Jauhar AA, Majid S, Ahmed V, Eisha A, Zaman N.M. 2006. Relationship between different allogamic associated trait characteristics of the five newly developed cytoplasmic male sterile (CMS) lines in rice. J. Cent. Eur. Agric. 7(1): 49-56. 
Ali SS, Khan MG. 1995. Maintainers and restorers identified from local germplasm in Pakistan using IRRI cytoplasmic male sterile lines. Intl. Rice Res. Notes. 20(2): 6.

Annual Report of Bangladesh Rice Research Institute 2010, Gazipur 1701, Bangladesh. 392p.

Borkakati RP, Chetia SK. 2000. Identification of parental lines for heterosis breeding in rice for Assam. J. Interacademica 4(2): 224-229.

Ganesan KN, and Rangaswamy M. 1998. Combining ability studies in rice hybrids involving wild abortive (WA) and Oryza perennis sources of CMS lines. Oryza. 35(2): 113116.

GovindaRaj K, Virmani SS. 1988. Genetics of fertility restoration of 'WA' type cytoplasmic male sterility in rice. Crop Sci. 28: 787-792.

GovindaRaj K, Virmani SS. 1989. Maintainer and restorers for different cytoplasmic male sterile systems. Intl. Rice Res. Newsl. 14(5): 7-8.

Hawlader MSH, Mian MAK, Ali M. 1997. Identification of male sterile sterility maintainer lines for Ogura radish (Raphanus sativa L.). Euphytica 96(2): 297-300.

Hoan NT, Sarma NP, Siddiq EA. 1998. Wide hybridization for diversification of CMS in rice. Intl. Rice Res. Notes. 23(1): 5-6.

Ingale BV, Waghmode BD, Hodawadekar SS. 2008 Identification of restorer and maintainers for different CMS lines of rice. Madras Agric. J. 95 (7-12): 266-277.

Jayamani P, Ganesan KN, Thyiyagarajan K, Rangaswamy M, Rangasamy P. 1998. A new CMS line with a wide compatibility gene. Intl. Rice Res. Notes. 23(1): 5.

Jayasudha S, Sharma D. 2010. Identification of restorers and maintainers for CMS lines of rice (Oryza sativa L.) under shallow low land condition. Electronic J. Plant Breed. 1(3): 311-314.

Julfiquar AW, Hasan JM, Azad AK, Nurunnabi AM. 2002. Research and Development of Hybrid Rice in Bangladesh: A Motivation to Adoption. In: Proc. 1st Natl. Workshop on Res. and Dev. of Hybrid rice in Bangladesh, Progress and Future Strategies held at Bangladesh Rice Research Institute on 5-6 January, 2002. 9-19pp.
Kaul MLH. 1997. Male sterility: cytological, biological and molecular alterations. In: Plant Breeding and Crop Improvement. Vol. II. Eds. Kapoor and Saini) CBS Publishers Distribution, New Delhi. 377-378pp.

Kumar RV, Satyanarayana PV, Rao MS. 1996. New cytoplasmic male sterile lines developed in Andhra Pradesh, India. Intl. Rice Res. Notes. 21(2-3): 30.

Kumari SL, Pattambi M, Dharwad, Kulkarni RS. 1998. Fertility restoration studies in four WA CMS lines of rice. Intl. Rice Res. Notes. 23(1): 9.

Rothschild GH. 1997. Hybrid Rice Breeding Manual, IRRI, Los Banos, Laguna, Phillippines.

Singh DK, Rajesh S, Singh R. 2000. Identification of parental lines for rice hybrids at varanasi location. Crop Res. Hisar. 20(2): 201-205.

Singh RK, Lal JP. 2005. Exploitation of heterosis in aromatic rices for different physico-chemical traits. Indian J. Genet. Pl. Breed. 65(1): 47-48.

Singh RV, Maurya DM, Dwivedi JL, Verma OP. 2005. Combining ability studies on yield and its components using CMS lines in rice (Oryza sativa L.). Oryza. 42: 306-309.

Teng LS, Shen ZT. 1994. 16. Inheritance of fertility restoration for cytoplasmic male sterility in rice. Rice Genet. Newsl. 11: 95-97.

Umadevi M, Veerabadhiran P, MBRRImani S, Shanmugasundaram P. 2010. Identification of potential maintainers and restorers using cytoplasmic male sterile lines in rice. Electronic J. Plant Breed. 1(4): 948-952.

Virmani SS, Viraktamath BC, Casal CL, Toledo RS, Lopez MT, Manalo JO. 1997. Hybrid Rice Breading Manual. Int. Rice Research Institute. Los Banos, Leguna, Philippines. 155p.

Zaman FU, Abraham MJ, Natarajan A, Mahendru A, Mohammad F. 1998. Developing Pusa 3A, a Basmati CMS line. Intl. Rice Res. Notes. 23(1):13. 